\title{
A ${ }^{12}$ CO Survey of the LMC with NANTEN
}

Y. Fukui, R. Abe, A. Hara, T. Hayakawa, S. Kato, A. Kawamura, A. Mizuno, N. Mizuno, H. Ogawa, T. Onishi, H. Saito, K. Tachihara, K. C. Xiao, N. Yamaguchi, and R. Yamaguchi

Department of Astrophysics, Nagoya University, Chikusa-ku, Nagoya, 464-8602, Japan

\section{Y. Yonekura}

Earth and Life Sciences, Osaka Prefecture University, Gakuencho 1-1, Sakai, Osaka 599-8531, Japan

\section{Rubio}

Departamento de Astronomia, Universidad de Chile, Casilla 36-D, Santiago, Chile

\begin{abstract}
We have made a ${ }^{12} \mathrm{CO}(J=1-0)$ survey of the LMC with NANTEN. A sample of 55 giant molecular clouds has been identified and comparisons with stellar clusters, HII regions and SNRs are presented. The connection between the clouds and cluster formation is discussed.
\end{abstract}

\section{Introduction}

The Large Magellanic Cloud (LMC) classified as the barred sub-type of Hubble's irregular class is the nearest neighbor to our own. Studies of this galaxy have provided invaluable information for our understanding of the universe and galaxies in various aspects including evolution of stars and stellar clusters, owing to its proximity to the solar system $(D \sim 50 \mathrm{kpc})$. Compared to the advanced evolutionary phase of stars, the process of star formation in the LMC has been only poorly understood, mainly due to the lack of comprehensive observations of giant molecular clouds where stars form. Previous observations of molecular gas in the LMC are either of low angular resolution or of small spatial coverage (Cohen et al. 1988; Israel et al. 1993; Kutner et al. 1997; Johansson et al. 1998). We have performed new observations of the LMC in the $J=1-0$ rotational transition of interstellar carbon monoxide $(\mathrm{CO})$ at $2.6 \mathrm{~mm}$ wavelength in order to reveal detailed molecular gas distribution at a linear resolution of $\sim 30 \mathrm{pc}$. These observations were made with NANTEN newly installed at Las Campanas Observatory, Chile in 1996 (Fig. 1) and should allow us to have a complete view of the giant molecular clouds in the LMC for the first time. Four related contributions included in these proceedings are complementary to this paper, describing more details of these $\mathrm{CO}$ observations and comparisons with other astronomical objects (Abe et al.; Mizuno et al.; Saito et al.; Yamaguchi et al.). 


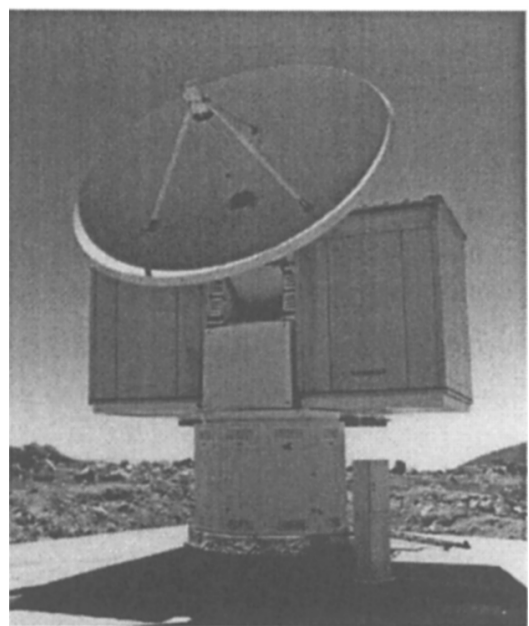

Figure 1. $4 \mathrm{~m}$ millimeter-wave telescope, NANTEN, of Nagoya University located at Las Campanas Observatory, Chile.

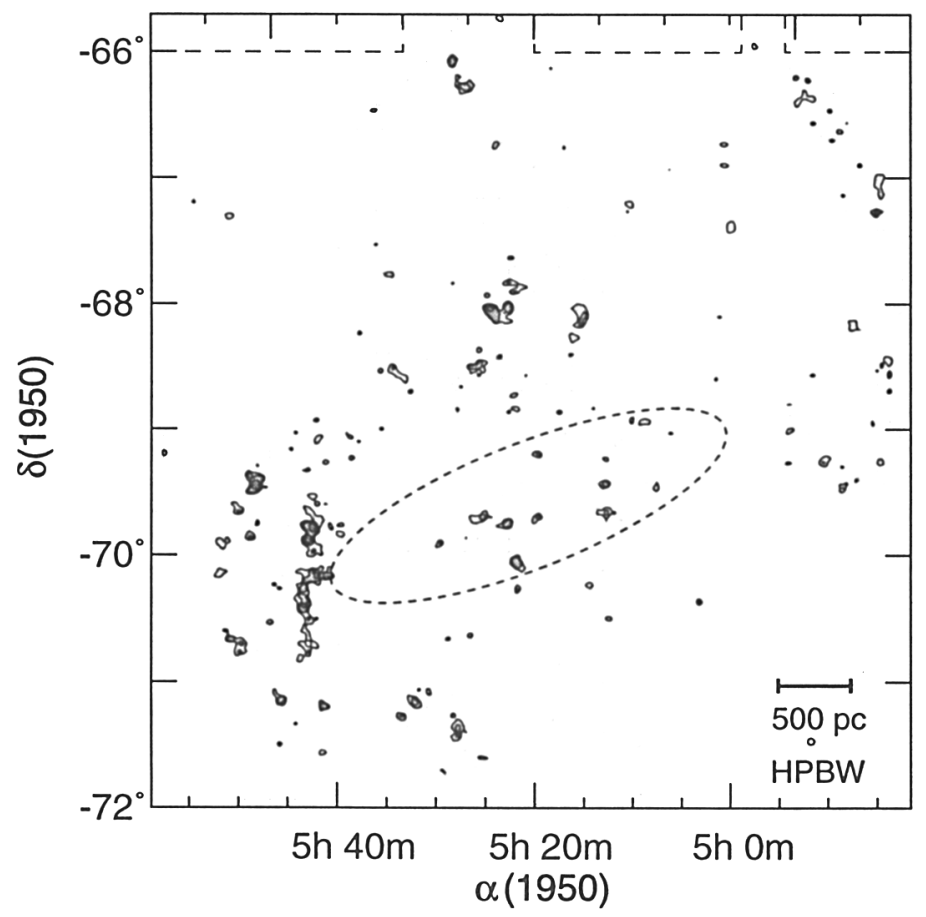

Figure 2. Velocity-integrated intensity of CO $(J=1-0)$ emission obtained with NANTEN. The lowest contour and the separations between contours are $3.0 \mathrm{~K} \mathrm{~km} \mathrm{~s}^{-1}$ for each. The dotted line shows the optical boundary of the Bar. 

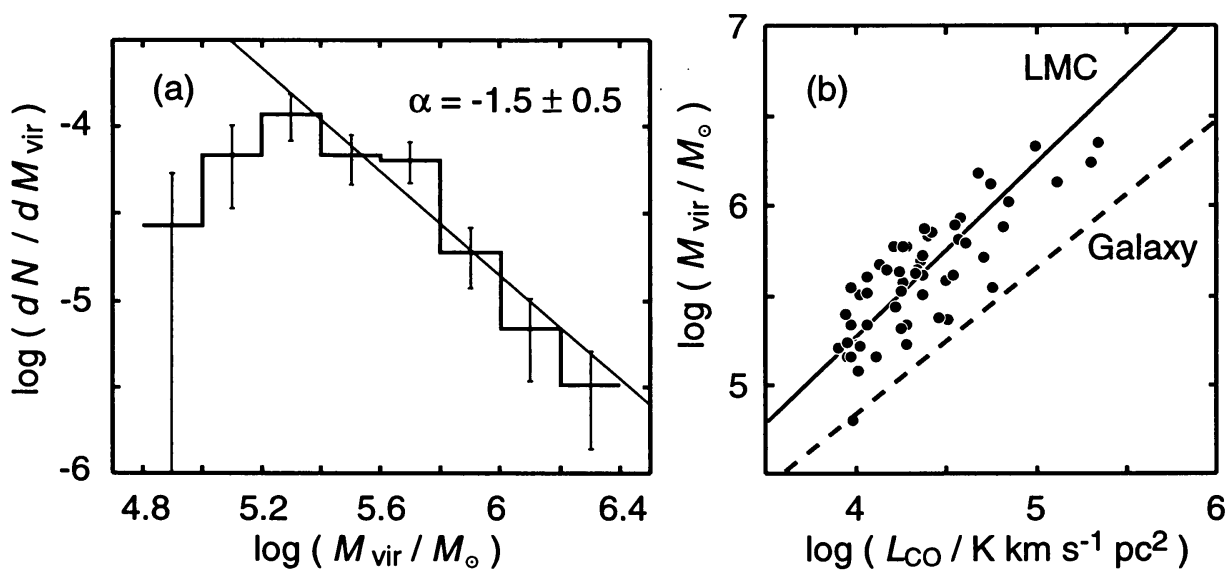

Figure 3. (a) Mass spectrum, $d N / d M_{\text {vir }}$, of the 55 clouds. The error bars represent the $(d N)^{1 / 2}$ statistical errors. The straight line is the least-squares fit to the clouds with $M_{\text {vir }} \geq 1.6 \times 10^{5} M_{\odot}$ (by $\left[d N / d M_{\text {vir }}\right]$ $=1.2 \times 10^{4}\left[M_{\text {vir }} / M_{\odot}\right]^{-1.5}$ ). (b) Virial mass, $M_{\text {vir }}$, plotted against CO luminosity, $L_{\mathrm{CO}}$. The solid line represents the least-square fit and the dashed line indicates the same relation but derived for the Galactic molecular clouds (Solomon et al. 1987).

\section{Properties of the Giant Molecular Clouds}

Figure 2 shows the CO image obtained with NANTEN. This image consists of 32,800 spectra taken at every $2^{\prime}$, corresponding to $\sim 30 \mathrm{pc}$ at a distance of $50 \mathrm{kpc}$, with a $2 ! 6$ beam. The distribution is highly clumpy, extended over 36 square-degrees. Large $\mathrm{CO}$ complexes whose size is more than $100 \mathrm{pc}$ are located at the southern part of 30 Dor and N44 regions. Smaller CO clouds are distributed over the observed area, with moderate concentration toward the Bar and toward several prominent HII regions. We note that an arclike semi-circular $\mathrm{CO}$ distribution whose diameter is $\sim 3 \mathrm{kpc}$ is seen in the south-east boundary of the optical galaxy.

The number of the $\mathrm{CO}$ clouds in Figure 2 is more than 100. Among them, there are $55 \mathrm{CO}$ clouds which are detected at more than three positions. The mass of these 55 clouds ranges from $\sim 10^{5} M_{\odot}$ to $\sim 3 \times 10^{6} M_{\odot}$, indicating that they are giant molecular clouds. In Figure $3 \mathrm{a}$, we present the mass spectrum $d N / d M_{\text {vir }}$ of the 55 clouds. It is fitted by a single power-law of an index of -1.5 in a cloud mass range $10^{5}-10^{6} M_{\odot}$, which is similar to that found in our Galaxy (Solomon et al. 1987). The lower boundary, $\simeq 10^{5} M_{\odot}$, is the present detection limit in mass. Figure $3 \mathrm{~b}$ shows the relation between the virial mass $\left(M_{\mathrm{vir}}\right)$ and CO luminosity $\left(L_{\mathrm{CO}}\right)$, for the $55 \mathrm{CO}$ clouds. The CO luminosities in the LMC are weaker by a factor of $\sim 3$ relative to those in the Galaxy. By using this factor, the total molecular cloud mass is estimated from the CO luminosities to be $\sim 4 \times 10^{7} M_{\odot}, 90 \%$ of which is included in the large 55 clouds whose $\mathrm{H}_{2}$ column density is greater than $2 \times 10^{21} \mathrm{~cm}^{-2}$. According to the subsequent, 
more sensitive observations of selected regions, the cloud mass may increase up to $\sim 7 \times 10^{7} M_{\odot}$ at the $\mathrm{H}_{2}$ column density $\geq 1 \times 10^{21} \mathrm{~cm}^{-2}$ (see Mizuno et al. 1999 in these proceedings). We, therefore, estimate the molecular mass to be 4 $-7 \times 10^{7} M_{\odot}$, corresponding to $5-10 \%$ of the $\mathrm{HI}$ mass $M(\mathrm{HI}) \sim 7 \times 10^{8} M_{\odot}$ (McGee \& Milton 1966).

The apparent weakness of $\mathrm{CO}$ relative to $M_{\text {vir }}$ (Fig. $3 \mathrm{~b}$ ) is possibly caused by the lower metal abundance and by enhanced photodissociation due to relatively strong UV radiation fields under significantly smaller shielding. The conversion factor $X \equiv N\left(\mathrm{H}_{2}\right) / I_{\mathrm{CO}}$, the ratio of $\mathrm{H}_{2}$ column density to $\mathrm{CO}$ line intensity, varies strongly with such physical conditions in clouds. The conversion factor, $X$, for clouds in the LMC, determined by assuming that they are virialized, is $9 \times 10^{20} \mathrm{~cm}^{-2} /\left(\mathrm{K} \mathrm{km} \mathrm{s}^{-1}\right)$. This is smaller than the value from Cohen et al. (1988) obtained with the $1.2 \mathrm{~m}$ radio telescope, but about 3 times higher than the value for our Galaxy (Solomon et al. 1987).

\section{Formation of Star Clusters}

In order to study star formation in the LMC, detailed comparisons of the CO clouds with stellar clusters, HII regions, and SNRs have been made over the whole Cloud as shown in Fig. 4.

The following are the results of the comparison of $\mathrm{CO}$ clouds with stellar clusters and $O B$ associations (hereafter, clusters). Out of the $55 \mathrm{CO}$ clouds, 26 are associated with clusters. Using the age of the clusters estimated from their color indices, UBV (Bica et al. 1996), $\sim 90 \%$ of the clusters associated with the $\mathrm{CO}$ clouds are younger than $10 \mathrm{Myr}$ (SWB0). The older clusters with $\tau>10$ Myr (SWBII-VII) show little correlation with the CO clouds. The youngest clusters associated with massive $\mathrm{CO}$ clouds tend to be in a compact group of young stellar clusters, i.e., N159 and N44. These groups of clusters are located at or near the peak of the $\mathrm{CO}$ clouds, indicating that they have been just formed in massive $\mathrm{CO}$ clouds.

The youngest clusters show a significant correlation with the $\mathrm{CO}$ clouds; about $\sim 30 \%$ of them are associated with CO clouds. The remaining $\sim 70 \%$ of the youngest clusters tend to be scattered, away from the $\mathrm{CO}$ clouds. Though these clusters may be associated with clouds smaller than the present detection limit, this suggests that young stellar clusters can rapidly dissipate their surrounding $\mathrm{CO}$ clouds (see also Yamaguchi et al. in these proceedings.)

Comparisons of the 55 massive $\mathrm{CO}$ clouds with HII regions and with stellar clusters indicate the following:

1. $12 \mathrm{CO}$ clouds show no sign of star formation; i.e., they are associated with no HII regions or stellar clusters.

2. $17 \mathrm{CO}$ clouds are associated with small HII regions only, but with no stellar clusters.

3. $26 \mathrm{CO}$ clouds are associated with stellar clusters and large HII regions, suggesting active, on-going formation of massive clusters (e.g., N44). 


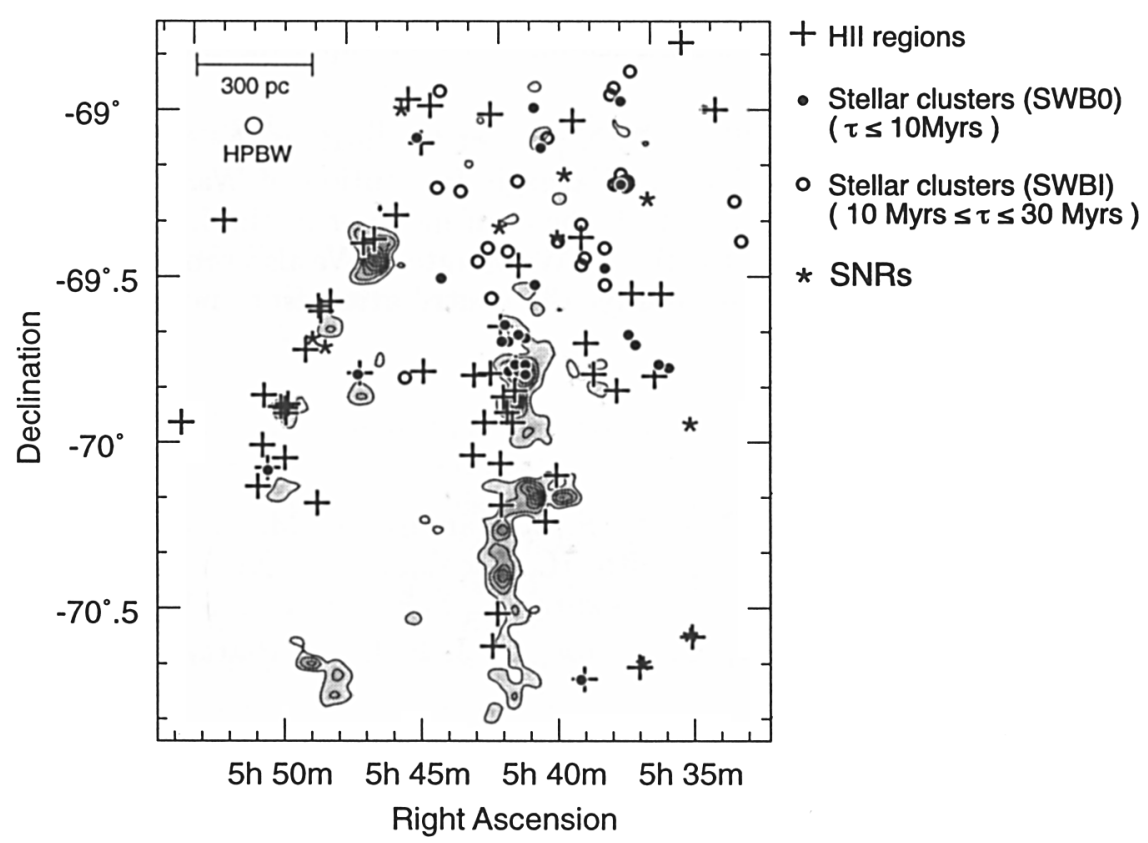

Figure 4. Distribution of the CO clouds, HII regions, stellar clusters and SNRs toward 30 Dor. The contours represent an intensity map of $\mathrm{CO}$; the lowest contour and the separations are each $3.0 \mathrm{~K} \mathrm{~km} \mathrm{~s}^{-1}$. The pluses indicate HII regions cataloged by Davies et al. (1976). The filled circles and squares are stellar clusters with $\tau \leq 10^{6}$ yr (SWB0) and $10^{6} \mathrm{yr} \leq \tau \leq 3 \times 10^{6} \mathrm{yr}$ (SWBI), respectively (Bica et al. 1996). The asterisks are SNRs (Mathewson et al. 1983).

On the other hand, there are several regions where stellar clusters (SWB0 and SWBI) are coexistent with large HII regions, but associated with only a small amount of $\mathrm{CO}$ clouds. Such an example is seen toward 30 Dor.

If we assume that the $\mathrm{CO}$ clouds in the LMC are being formed nearly steadily, the above comparisons may represent evolution of the $\mathrm{CO}$ clouds. The fact that about a half of the CO clouds are associated with the youngest clusters, SWB0, suggests that stellar clusters are activey formed over $\sim 50 \%$ of a cloud's lifetime. This lifetime is roughly estimated to be $\sim 3 \mathrm{Myr}$, since $\sim 1 / 3$ of the SWB0 clusters, which are younger than $10 \mathrm{Myr}$, are associated with CO clouds. By including the period prior to cluster formation, the typical lifetime of a $\mathrm{CO}$ cloud may be estimated as $\sim 6 \mathrm{Myr}$ the LMC. On the other hand, the absence of a massive CO cloud near the rest of the SWB0 clusters suggests that cloud dissipation is fairly rapid, possibly due to stellar UV photons (see Yamaguchi et al. in these proceedings).

To summarize, the complete sample of the giant molecular clouds in a single galaxy, the LMC, has allowed us to pursue how formation of stellar clusters takes place in them. The present results suggest that a giant molecular cloud forms 
massive stellar clusters fairly quickly, leading to very rapid dissipation of itself over a timescale of some $6 \mathrm{Myr}$.

Acknowledgments. The NANTEN project is based on the mutual agreement between Nagoya University and Carnegie Institution of Washington. We greatly appreciate the hospitality of all the staff member of the Las Campanas Observatory of the Carnegie Institution of Washington. We also proudly mention that this project has been supported by the contribution from many Japanese public donators and companies.

\section{References}

Abe, R., Hara, A., Hayakawa, T., Kato, S., Kawamura, A., Mizuno, A., Mizuno, N., Ogawa, H., Onishi, T., Saito, H., Tachihara, K., Xiao, K. C., Yamaguchi, N., Yamaguchi, R., Yonekura, Y., \& Fukui, Y. 1999, this volume

Bica, E., Claria, J. J., Dottori, H., Santos, Jr. J. F. C., \& Piatty, A. E. 1996, ApJS, 102, 57

Cohen, R. S., Dame, T. M., Garay, G., Montani, J., Rubio, M., \& Thaddeus, P. 1988, ApJ, 331, L95

Davies, R. D., Elliott, K. H., \& Meaburn, J. 1976, MmRAS, 81, 89

Israel, F. P., Johansson, L. E. B., Lequeux, J., Booth, R. S. , Nyman, L.A., Crane, P., Rubio, M., de Graauw, Th., Kutner, M. L., Gredel, R., Boulanger, F., Garay, G., \& Westerlund, B. 1993, A\&A, 276, 25

Johansson, L. E. B., Greve, A., Booth, R. S., Boulanger, F., Garay, G., de Graauw, Th., Israel, F. P., Kutner, M. L., Lequeux, J., Murphy, D. C., Nyman, L.-A., \& Rubio, M. 1998, A\&A, 331, 857

Kutner, M. L., Rubio, M., Booth, R. S., Boulanger, F., de Graauw, Th., Garay, G., Israel, F. P., Johansson, L. E. B., Lequeux, J., \& Nyman, L.-A. 1997, A\&AS, 122, 255

Mathewson, D. S., Ford, V. L., Dopita, M. A., Tuohy, I. R., Long, K. S., \& Helfand, D. J. 1983, ApJS, 51, 345

McGee, R. X., \& Milton, J. A. 1966, Austr. J. Phys. 19, 343

Mizuno, N., Abe, R., Hara, A., Hayakawa, T., Kato, S., Kawamura, A., Mizuno, A., Ogawa, H., Onishi, T., Saito, H., Tachihara, K., Xiao, K. C., Yamaguchi, N., Yamaguchi, R., Yonekura, Y., \& Fukui, Y. 1999, this volume

Saito, H., Abe, R., Hara, A., Hayakawa, T., Kato, S., Kawamura, A., Mizuno, A., Mizuno, N., Ogawa, H., Onishi, T., Tachihara, K., Xiao, K. C., Yamaguchi, N., Yamaguchi, R., Yonekura, Y., \& Fukui, Y. 1999, this volume

Solomon, P. M., Rivolo, A. R., Barrett, J., \& Yahil, A. 1987, ApJ, 319, 730

Yamaguchi, R., Abe, R., Hara, A., Hayakawa, T., Kato, S., Kawamura, A., Mizuno, A., Mizuno, N., Ogawa, H., Onishi, T., Saito, H., Tachihara, K., Xiao, K. C., Yamaguchi, N., Yonekura, Y., \& Fukui, Y. 1999, this volume 